\title{
Point-of-care haemostatic management in orthotopic liver transplantation of an ex situ hepatectomized liver graft
}

Katsanoulas K., Katsika E, Georgopoulou E., Markopoulos I., Serchan P., Bali E. Hippokrateion General Hospital, Anaesthesiology Dept., Thessaloniki, Greece

\section{BACKGROUND}

Anatomical considerations in split-liver transplantation
$>$ Variant hepatic anatomy must be recognized and managed
during split-liver transplantation (SLT).
In SLT, portal flow is redirected through relatively
small-for-size (SFS) grafts, leading to SFS syndrome (SFSS)
Good graft function depends on graft's intact arterial and
portal supply as well as biliary and venous drainage from
all retained liver segments.
Liver splitting in the heart-beating donor, abolishes ex situ
benching and prolonged ischemia time and provides two
anatomically perfect grafts with hemostasis accomplished.

\section{Challenges in haemostatic management in OLT \\ $>$ Reduced levels of both procoagulant factors and inhibitors. \\ $>$ Thrombocytopenia/dysfunction is counterbalanced by the increased levels of fVIII and vWf. \\ $>$ Hyperfibrinolysis is a major issue after reperfusion. \\ $>$ ESA 2013: "We recommend the use of perioperative ROTEM/TEG monitoring for targeted management of coagulopathy", \\ $>$ Goal-directed P-O-C parameters algorithmic approach.}

\section{CASE REPORT}

* Male, 45 y, BMI $30 \mathrm{~kg} / \mathrm{cm}^{2}$

* Alcoholic Cirrhosis MELD score 16

* Four HE episodes, type II diabetes

*oagulation profile : INR 1,8 PT 19,9s APTT 50,7s

FIB $1,94 \mathrm{~g} / \mathrm{dl}$ PLTs $105000 \mu / 1$

* No signs of HE, varices or pulmonary hypertension

- Spironolactone, furosemide, lactulose, $\mathrm{Mg}$, folic acid, vitB

\section{Graft peculiarity (figures)}

*xceptional left liver lobe perfusion.

Ectopic hepatic artery rising directly from the aorta (rare anatomic variation, 1.6\%).

* Vessel endothelium "fractured".

* Extended graft hepatectomy of the depended liver segments (II and III) on the bench.

* Extended surface with no ex vivo surgical haemostasis.
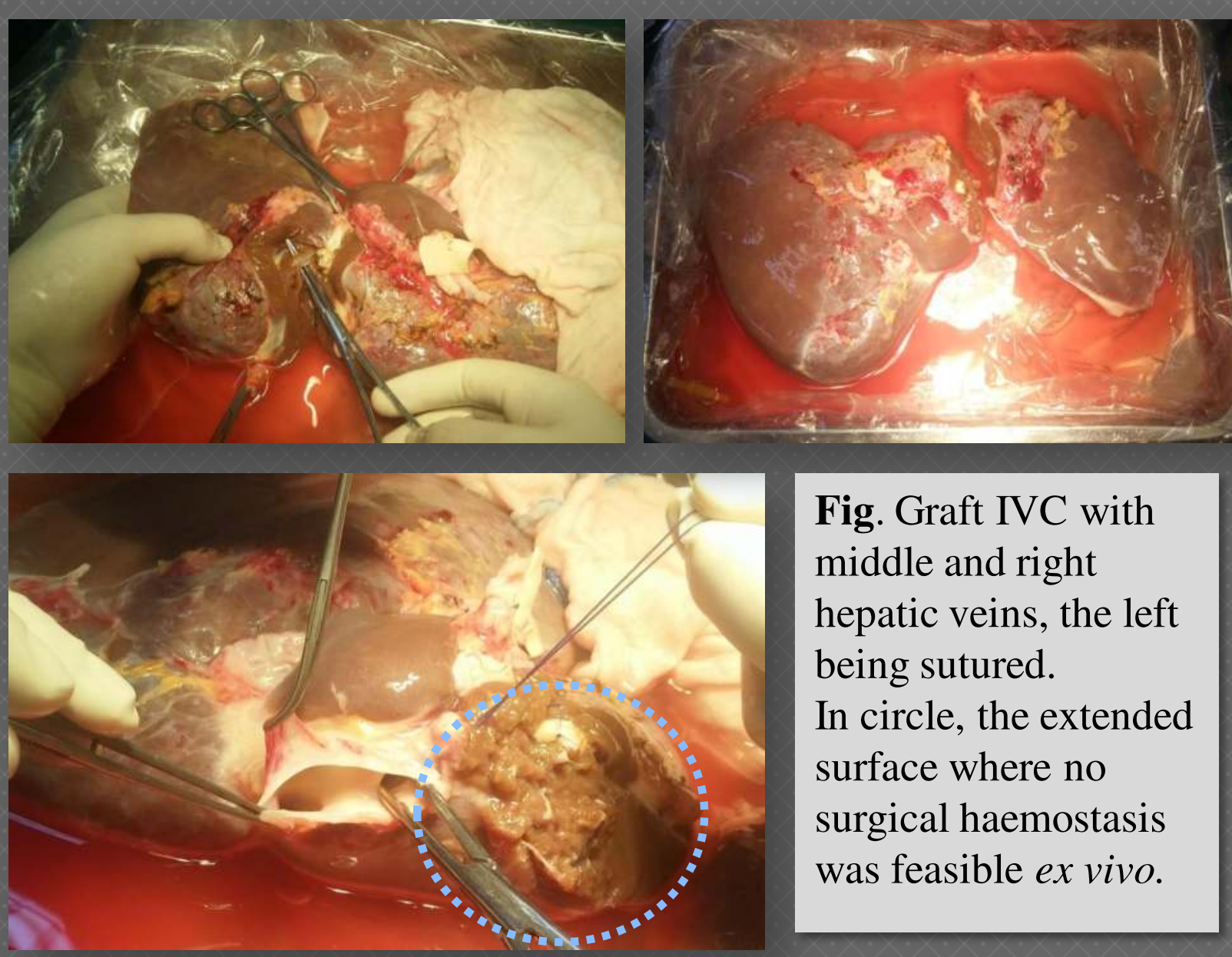

Fig. Graft IVC with middle and right hepatic veins, the left being sutured. In circle, the extended surface where no surgical haemostasis was feasible ex vivo.
Orthotopic liver transplantation (piggy pack technique)

Hbg 10.5 (start) - 9.3 (end) g/dl, 2 PRBCs after reperfusion

* Fluids balance $+6000 \mathrm{ml}$

* 10 hrs cold ischemia time, anhepatic phase $2.5 \mathrm{hrs}$

uneventful reperfusion, Lactate $_{\max } 7 \mathrm{mmol} / \mathrm{L}$

* Total OLTx duration 6 hrs

* Acceptable haemodynamics CI 3.2-5.0, PVRI 139-92,

$\mathrm{Ca}-\mathrm{vO}_{2} 2.7-2.9, \mathrm{SvO}_{2} 81-72 \%$

* Prolonged ICU stay, mild cholestasis but no signs of SFSS.

* Discharged tracheostomized but in good general state.

\section{DISCUSSION}

Haemostatic management and transfusions were directed by ROTEM® following a published algorithm.

(Goerlinger K, 2006, rev. 2014).

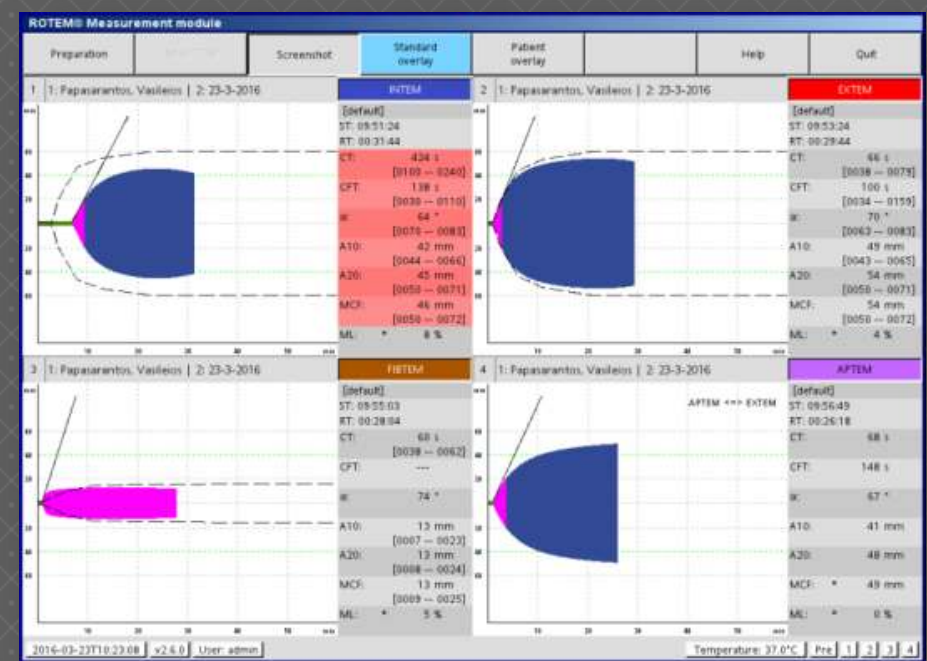

PREANHEPATIC PHASE No bleeding

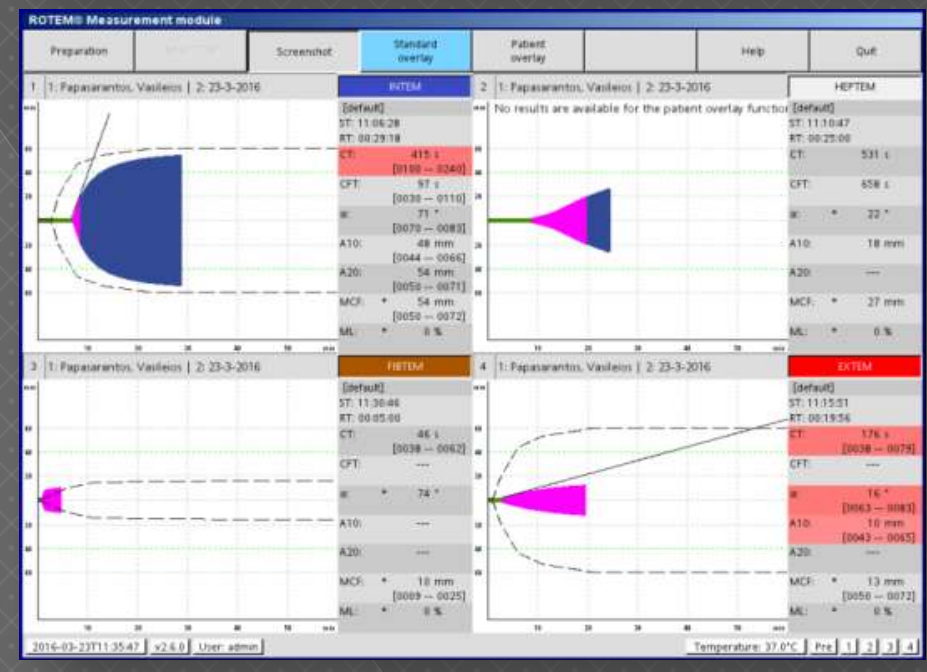

\begin{tabular}{|c|c|}
\hline $\mathrm{CT}_{\text {EXTEM }}$ & $\mathrm{A5}_{\text {EXTEM }}$ \\
\hline 66 & 49 \\
\hline $\mathrm{A} 5_{\text {FIBTEM }}$ & $\mathrm{MCF}_{\text {EXTEM }}$ \\
\hline 13 & 54 \\
\hline
\end{tabular}

ANHEPATIC PHASE Mild diffuse bleeding

\begin{tabular}{|c|c|}
\hline CT $_{\text {EXTEM }}$ & A5 EXTEM $_{\text {EIEM }}$ \\
\hline 176 & 7 \\
\hline A5 $_{\text {FIBTEM }}$ & MCF $_{\text {EXTEM }}$ \\
\hline 11 & 14 \\
\hline
\end{tabular}

$\checkmark 1500$ U Prothrombin Complex Concentrate, (Beriplex®)

$\checkmark$ No Platelet transfusion (although considered)

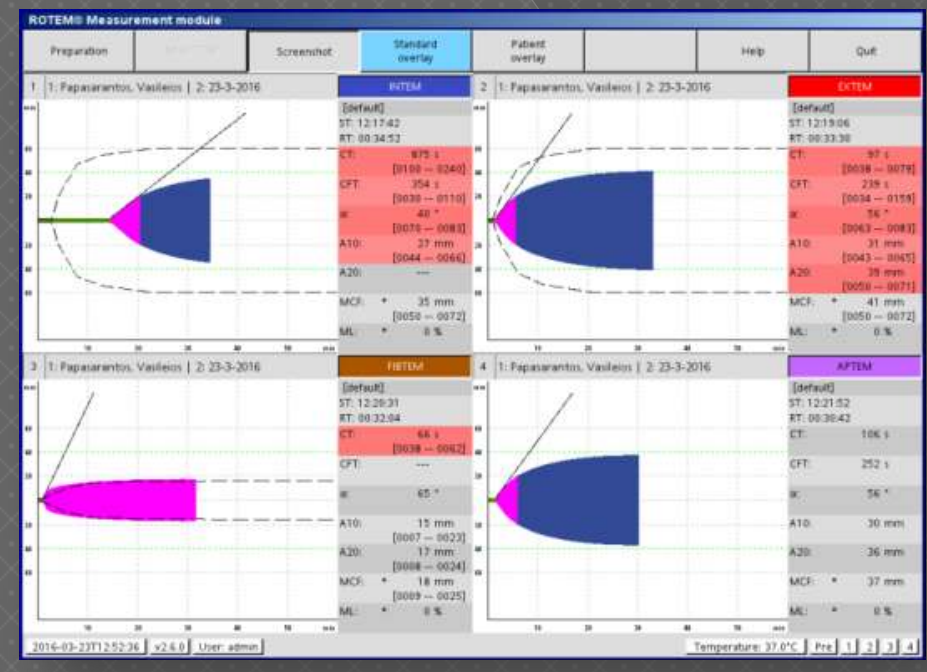

NEOHEPATIC PHASE

Minimal bleeding

\begin{tabular}{|c|c|}
\hline $\mathrm{CT}_{\text {EXTEM }}$ & $\mathrm{A5}_{\text {EXTEM }}$ \\
\hline 97 & 23 \\
\hline $\mathrm{A} 5_{\text {FIBTEM }}$ & $\mathrm{MCF}_{\text {EXTEM }}$ \\
\hline 13 & 41 \\
\hline
\end{tabular}

$\checkmark$ No Platelet transfusion (although considered)

$\checkmark 1 g$ Fibrinogen conc. (Riastap®) to enhance clot strength $\checkmark$ Bleeding ceased completely

\section{LEARNING POINTS}

Point-of-care haemostatic management combined with close clinical evaluation of surgical bleeding contributed successfully to safe decision making in this complex case.

REFERENCES

- Goss JA et al. In situ splitting of the cadaveric liver for transplantation. Transplantation. 1997 Sep 27;64(6):871-7.

- Chaib E. et al. The main hepatic anatomic variations for the purpose of split-liver transplantation. Transplant Proc. 2005 Mar;37(2):1063-6.

- Goerlinger K. Coagulation management during liver transplantation.

Hämostaseologie 2006; 26 (Suppl 1): S 64 -S 75 